\title{
Influence of Oxidants on the Stability of Tocopherol in Model Nanoemulsions: Role of Interfacial Membrane Organized by Nonionic Emulsifiers
}

\author{
Jinhyuk Kim, ${ }^{1}$ Ha Youn Song, ${ }^{2}$ and Seung Jun Choi $\mathbb{D I}^{1,3}$ \\ ${ }^{1}$ Department of Food Science and Technology, Seoul National University of Science and Technology, Seoul 01811, \\ Republic of Korea \\ ${ }^{2}$ Department of Agricultural Biotechnology, Seoul National University, Seoul 08826, Republic of Korea \\ ${ }^{3}$ Department of Interdisciplinary Bio IT Materials, Seoul National University of Science and Technology, Seoul 01811, \\ Republic of Korea \\ Correspondence should be addressed to Seung Jun Choi; choisj@seoultech.ac.kr
}

Received 17 August 2018; Accepted 16 October 2018; Published 12 November 2018

Guest Editor: Hiroshi Umakoshi

Copyright (C) 2018 Jinhyuk Kim et al. This is an open access article distributed under the Creative Commons Attribution License, which permits unrestricted use, distribution, and reproduction in any medium, provided the original work is properly cited.

\begin{abstract}
Nanoemulsions were prepared by using emulsifiers with various sizes of hydrophilic and hydrophobic groups to determine the impact of interfacial characteristics on the stability of $\alpha$-tocopherol incorporated into the nanoemulsions. The $\alpha$-tocopherol concentration remaining after 3 weeks of storage at $25^{\circ} \mathrm{C}$ depended greatly on the type of oxidative stress, which indicated that the environment surrounding the oil droplets could determine the stability of $\alpha$-tocopherol in nanoemulsions. $\alpha$-Tocopherol was gradually degraded by radical-mediated oxidation over storage, and approximately $60 \%$ of its initial concentration remained after 3 weeks of storage. However, under acid- and iron-mediated oxidation, $\alpha$-tocopherol concentration steeply decreases for the initial 3-day storage, but the degradation rate of $\alpha$-tocopherol decreased after 3 days of storage and over $90 \%$ of the initial $\alpha$-tocopherol remained after 3 weeks of storage. Interestingly, and contrary to our expectations, the thickness and/or density of the droplet interfacial membrane rarely affected the stability of $\alpha$-tocopherol incorporated into nanoemulsions. Although it is difficult to generalize beyond $\alpha$-tocopherol, we conclude that the properties of oil droplet surfaces had no influence on the storage stability of $\alpha$-tocopherol encapsulated in the droplets.
\end{abstract}

\section{Introduction}

Lipid oxidation is one of the greatest concerns for oilcontaining food products because of its negative influence on nutritional quality and consumer health [1]. In addition to lipid oxidation, lipophilic functional compounds incorporated into emulsion-based delivery systems can be decomposed by various oxidative stresses [2]. To inhibit lipid oxidation and to prevent lipophilic compound decomposition, several synthetic and natural antioxidants are generally incorporated into foods containing considerable amounts of lipids [3]. However, as a result of consumers' demands for clean food (i.e., food products that do not contain synthetic additives), food manufacturers have been making various attempts to replace synthetic antioxidants with natural ones. Among the natural antioxidants permitted for food use, tocopherols are important because they exist naturally in many vegetable oils [4] and because they have the ability to retard lipid oxidation by reacting with several radicals generated from lipid molecules, thereby protecting functional compounds from oxidative degradation. $\alpha$-Tocopherol radicals can form nonradical products if they are reduced by other coexisting antioxidants, with regeneration of $\alpha$-tocopherol.

In food systems, oil-in-water emulsions generally consist of water and oil, with the oil being dispersed as small droplets in the water [5]. Emulsifiers have surface activity, so they can create kinetically stable emulsions by absorbing at the surfaces of droplets newly formed during homogenization [6]. Emulsifiers absorbed at the oil droplet surfaces 
create an interfacial membrane comprised of a layer formed of their hydrophobic tails and a layer formed of their hydrophilic heads. Physical destabilization processes, such as coalescence, flocculation, and Ostwald ripening, are greatly affected by the characteristics of interfacial membranes formed with emulsifiers [7]. Interfacial membranes also alter the rates of chemical reactions, such as lipid oxidation [8-10], between oil- and aqueous-phase compounds. Additionally, when a functional lipophilic compound is incorporated into emulsion droplets, the interfacial properties of the oil droplet surface are the main factors that control the stability of the functional compound incorporated therein $[3,11]$. Because the interfacial membrane is formed with emulsifiers, the structural and physicochemical properties of emulsifiers play important roles in the emulsion stability and in the storage stability of functional compounds incorporated into the oil droplets [12].

Oil-in-water $(\mathrm{O} / \mathrm{W})$ emulsions are widely used as delivery systems in a variety of industries because of their abilities to encapsulate functional lipophilic compounds. Generally, O/W emulsions are classified into conventional emulsions (usually called "emulsions") and nanoemulsions, according to the size of the emulsion droplets [13]. Because nanoemulsions have much larger specific surface areas than conventional emulsions, chemical degradation reactions at the oil-water interface can occur more quickly in nanoemulsions than in conventional emulsions [14]. Therefore, when $\mathrm{O} / \mathrm{W}$ nanoemulsions are used as nanocarriers in delivery systems, the interfacial membrane formed by the emulsifier is an important factor in controlling the ability of the emulsion to protect the encapsulated functional compounds and to inhibit their diffusion from the oil droplets into the aqueous phase.

Therefore, in nanoemulsion-based oral delivery systems for functional lipophilic compounds, it is important to know how the interfacial membrane affects the stability of $\alpha$-tocopherol incorporated into the emulsion for the prevention of lipid oxidation and to understand the effectiveness of $\alpha$-tocopherol in inhibiting the degradation of the functional lipophilic compounds by oxidative stress from the aqueous phase. Over the past decades, scientists have investigated how the stability and effectiveness of emulsion systems are altered during the incorporation of $\alpha$-tocopherol $[15,16]$. The charge of the emulsion droplet surfaces is one of the major factors influencing the oxidative stability of the emulsified oil and also the stability of the $\alpha$-tocopherol. The chemical stability of $\alpha$-tocopherol is effectively improved in emulsions droplets with positively charged surfaces. When $\alpha$-tocopherol is incorporated into an emulsion with negatively charged interfaces, the $\alpha$-tocopherol stability can be increased by adding a biopolymer layer with a positive charge to the negatively charged droplet surfaces. However, despite this, most studies on the influence of interfacial membrane properties have shown that the charge of the emulsion droplet surfaces is a key factor influencing the stability of $\alpha$-tocopherol in emulsions and in lipid oxidation. Therefore, the objective of this work was to determine whether the structural properties of the interfacial membrane could be involved in $\alpha$-tocopherol decomposition in emulsions, particularly emulsion-based delivery systems. This evaluation was accomplished by using model emulsionbased delivery systems stabilized by emulsifiers with different hydrophilic head sizes, which led to various droplet interfacial thicknesses.

\section{Materials and Methods}

2.1. Materials. Polyoxyethylene alkyl ether-type emulsifiers (polyoxyethylene 10 lauryl ether (P10L), polyoxyethylene 10 stearyl ether (P10S), polyoxyethylene 20 stearyl ether (P20S), and polyoxyethylene 23 lauryl ether (P23L), and polyoxyethylene 100 stearyl ether (P100S)) were purchased from Sigma-Aldrich (St. Louis, MO, USA). The molecular structures of the polyoxyethylene alkyl ether-type emulsifiers used in this study are presented in Figure 1. $\alpha$-Tocopherol, 2,2' azobis(2-methylpropionamidine)dihydrochloride (AAPH), ferrous sulfate heptahydrate $\left(\mathrm{FeSO}_{4} \cdot 7 \mathrm{H}_{2} \mathrm{O}\right)$, and ferric chloride hexahydrate $\left(\mathrm{FeCl}_{3} \cdot 6 \mathrm{H}_{2} \mathrm{O}\right)$ were also purchased from Sigma-Aldrich. Medium-chain triglyceride (Delios S) comprised caprylic (70\%) and 30\% capric (30\%) acids and were obtained from BASF (Ludwigshafen, Germany). All other chemicals used were of analytical grade.

2.2. Emulsion Preparation. The aqueous phase was prepared by dissolving the emulsifiers in the phosphate buffer $(10 \mathrm{mM}$ and $\mathrm{pH}$ 7) to a predetermined concentration, and the oil phase was prepared by dissolving $\alpha$-tocopherol in mediumchain triglycerides at a final concentration of $5 \mathrm{mmol} / \mathrm{kg}$. Coarse emulsions were prepared by homogenizing the oil $(5 \%, \mathrm{w} / \mathrm{w})$ and aqueous $(95 \%, \mathrm{w} / \mathrm{w})$ phases $(0.25 \mathrm{mmol}$ $\alpha$-tocopherol $/ \mathrm{kg}$ emulsion) in a high-speed blender (T18 Basic Ultra-Turrax, Ika, Staufen, Germany) for $2 \mathrm{~min}$ at room temperature. The oil droplet sizes in the coarse emulsions were then reduced with 5 passes through a microfluidizer (MN400BF, Micronox, Seongnam, Korea) at $100 \mathrm{MPa}$. After adjustment of the $\mathrm{pH}$ level of the emulsions to a predetermined value, the emulsions were purged with nitrogen with gentle stirring for $30 \mathrm{~min}$ before the subsequent step. To determine the effect of transition metals on $\alpha$-tocopherol stability in the emulsions, ferrous sulfate or ferric chloride solution was added to the emulsions at a final concentration of $1 \mathrm{mmol} / \mathrm{kg}$ emulsion. Furthermore, to evaluate the effect of free radicals on $\alpha$-tocopherol stability in the emulsions, AAPH solution was added to the emulsions to a final concentration of $1 \mathrm{mmol} / \mathrm{kg}$ emulsion. Then, $10 \mathrm{~g}$ of the emulsion sample were transferred into $12 \mathrm{~mL}$ of a glass vial, closed airtight, and were stored in the dark at $25^{\circ} \mathrm{C}$.

2.3. Droplet Size Measurement. The mean emulsion droplet diameters were measured by using static light scattering (laser diffraction). To avoid multiple scattering effects, all emulsion samples were diluted to a droplet concentration of approximately $0.005 \%(\mathrm{w} / \mathrm{w})$ with a buffer solution of the same $\mathrm{pH}$ value as the sample, and samples were stirred continuously throughout the measurements to ensure homogeneity. The refractive index values for MCT and buffer solution were set at 1.47 and 1.33, respectively. The particle 


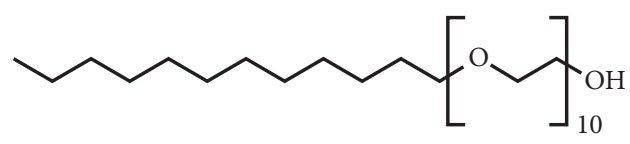

(a)

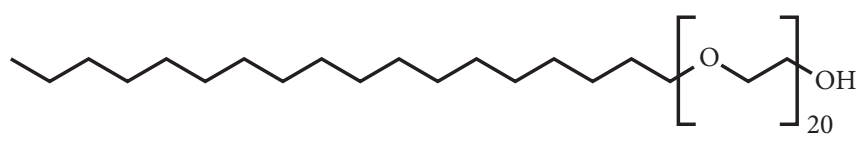

(c)

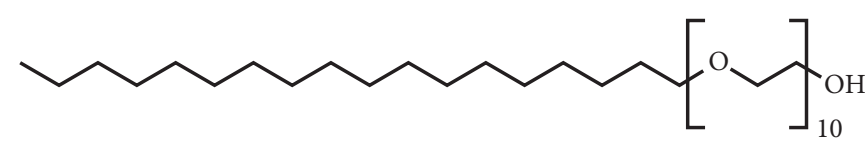

(b)

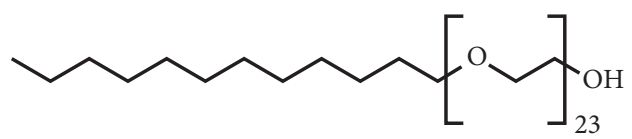

(d)

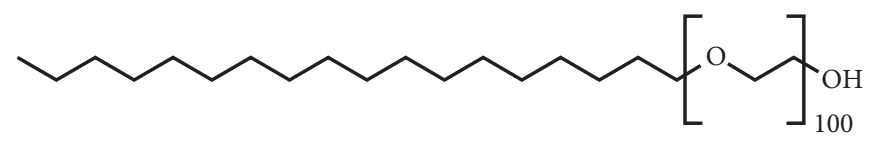

(e)

FIGURE 1: Molecular structures of polyoxyethylene alkyl ether-type emulsifiers used in this work. (a) polyoxyethylene 10 lauryl ether (P10L); (b) polyoxyethylene 10 stearyl ether (P10S); (c) polyoxyethylene 20 stearyl ether (P20S); (d) polyoxyethylene 23 lauryl ether (P23L); (e) polyoxyethylene 100 stearyl ether (P100S).

size distribution of the emulsions was then measured by using a commercial static light scattering instrument (BT9300ST; Bettersize Instruments, Dandong, China). The particle size data are reported as the volume-weighted mean diameter, $d_{43}=\sum n_{i} \cdot d_{i}^{4} / \sum n_{i} \cdot d_{i}^{3}$, with $n_{i}$ representing the number of particles with diameter $d_{i}$.

2.4. $\alpha$-Tocopherol Concentration Measurement. $\alpha$-Tocopherol concentration was measured according to the method described by Yang et al. [17] with slight modification. $\alpha$-Tocopherol concentrations in emulsions were determined by first vigorously vortexing $2 \mathrm{~g}$ of emulsion with $4 \mathrm{~g}$ of dichloromethane for $2 \mathrm{~min}$. The mixture was then centrifuged at $1,842 \times \mathrm{g}$ for $10 \mathrm{~min}$ at $25^{\circ} \mathrm{C}$, and the solvent layer was collected. $\alpha$-Tocopherol concentrations were determined with HPLC by using an Agilent 1100 instrument (Palo Alto, CA, USA). A Triart C18 column $(250 \mathrm{~mm} \times$ $4.6 \mathrm{~mm} \times 5 \mu \mathrm{m}$, YMC, Tokyo, Japan) was used, with a methanol mobile phase at a rate of $1 \mathrm{~mL} / \mathrm{min}$. The wavelength for detection was $295 \mathrm{~nm}$. Concentrations of $\alpha$-tocopherol were calculated on the basis of a calibration curve generated by using authentic $\alpha$-tocopherol.

Therefore, the decomposition rate $(k)$ of $\alpha$-tocopherol was calculated, by assuming a first-order reaction:

$$
C_{t}=C_{0} \cdot e^{-k \cdot t},
$$

where $C_{0}$ is the initial $\alpha$-tocopherol concentration ( $\mathrm{mmol} / \mathrm{kg}$ emulsion) and $C_{t}$ is the $\alpha$-tocopherol concentration remaining at time $t$ (day). The $k$ value was calculated by performing a linear regression on the plot $\ln \left(C_{t} / C_{0}\right)$ versus $t$. The equality of coefficients of different linear regressions was analyzed by the Chow test [18]. If time-dependent changes in the degradation of $\alpha$-tocopherol in emulsions were observed (the fast degradation of $\alpha$-tocopherol in the early stage of storage and the slow its degradation in the late storage period in this study), the initial $\alpha$-tocopherol decomposition rate $(\mathrm{mmol} / \mathrm{kg}$ emulsion/day) was determined. The initial $\alpha$-tocopherol decomposition rate could be determined by calculating the tangential slope at $t=0$ because the instantaneous rate at time $t$ is determined by calculating the tangential slope at $t$ on $\alpha$-tocopherol concentration versus time curve.

2.5. Statistical Analysis. All the experiments were performed in triplicate, and the data are expressed as mean \pm standard deviation. Analysis of variance (ANOVA) was performed, and the mean separations were performed using Duncan's multiple range test $(p<0.05)$. The statistical analyses described above were all conducted using SAS (version 9.4.; SAS Institute Inc., Cary, NC, USA).

\section{Results and Discussion}

To minimize the negative effect of micelles on the stability of the emulsions and $\alpha$-tocopherol therein [19], the minimum emulsifier concentrations (MECs) required to prepare highly stable emulsions with mostly small droplets were determined in our previous study [7]. The MECs for P10L, P10S, P20S, P23L, and P100S were 2.903, 3.165, 2.926, 1.784, and $0.994 \mathrm{mM}$, respectively. As all emulsions prepared at the MECs had similar initial droplet diameters $\left(d_{43}=0.29,0.28\right.$, $0.29,0.32$, and $0.28 \mu \mathrm{m}$ for P10L-, P10S-, P20S-, P23L-, and P100S-stabilized emulsions, respectively) and the droplet sizes rarely changed after 21 days of storage, and any significant difference in the $\alpha$-tocopherol decomposition rate between emulsions could not stem from an effect of the oil droplet interfacial area. Although the emulsifier concentration in emulsions were different from each emulsion, the facts that all emulsions had a similar oil droplet size indicated that emulsions had the different emulsifier loading (emulsifier concentration per unit droplet surface area) values which could be attributed to droplet interfacial density. The emulsifier loading values for P10L-, P10S-, P20S-, P23L-, and P100S-stabilized emulsions were calculated as $1.91,2.90,1.87,1.24$, and $0.62 \mu \mathrm{mol} / \mathrm{m}^{2}$, respectively, indicating that the interfacial density of emulsions differed. When oils are stabilized by emulsifiers to spherical droplets, the interfacial membrane of oil droplet surfaces are 
comprised of the inner layer formed with the hydrophobic tails of emulsifiers and the outer layer formed with their hydrophilic heads. Considering the molecular structures of emulsifiers used in this work, it means that the thickness of the outer layer of the interfacial membrane could be mainly attributed by a number of oxyethylene groups of the hydrophilic groups of emulsifiers and that the length of alkyl chains of the hydrophobic tails of emulsifiers could determine the thickness of its inner layer.

Since the partition coefficient of $\alpha$-tocopherol is approximately 12 [20], it is likely that the concentration of $\alpha$-tocopherol in the aqueous phase was negligible. Therefore, if $\alpha$-tocopherol degradation is observed after a certain period of storage and prooxidants are present in the aqueous phase, most of $\alpha$-tocopherol must have decomposed at the emulsion droplet surface, rather than in the aqueous phase.

\subsection{Influence of the $p H$ Level on $\alpha$-Tocopherol Degradation.} Vitamin E compounds, including $\alpha$-tocopherol, exhibit fairly good stability in the absence of oxygen and lipid peroxides [21]. However, with the consideration that commercially available emulsion-based foods are generally acidic [10] and molecular oxygen is never completely removed from them, it is important to understand the influence of the $\mathrm{pH}$ level on the chemical stability of $\alpha$-tocopherol in emulsions. Therefore, to examine how the characteristics of emulsion droplet surfaces affect the chemical decomposition of $\alpha$-tocopherol in acidic environments, the $\mathrm{pH}$ level of the emulsions was adjusted to 7 or 3 and the emulsions were then stored. Because mediumchain triglycerides consist of only saturated fatty acids, they are exceptionally stable to oxidation [22]. In addition, most of the oxygen molecules were removed by nitrogen purging. Therefore, if $\alpha$-tocopherol degradation is observed to a considerable level, it could be the result of factors other than lipid peroxides derived from the oxidation of the medium-chain triglyceride carrier oil. One possible reason for the reduction of $\alpha$-tocopherol during storage is the presence of a trace amount of oxygen molecules in the emulsions. In this study, to minimize the effect of oxygen molecules on $\alpha$-tocopherol decomposition, nitrogen purging was carried out to remove oxygen molecules. However, it seems that the oxygen molecules in the aqueous phase were not completely removed. The effects of the emulsifier and $\mathrm{pH}$ level on $\alpha$-tocopherol stability in emulsions are shown in Figure 2. As indicated in Table 1, regardless of the $\mathrm{pH}$ level, P100S-stabilized emulsions showed the highest initial decomposition rate of $\alpha$-tocopherol among the emulsions. P100S has the largest hydrophilic head size among the emulsifiers used and the P100S-stabilized emulsion contained the smallest amount of the emulsifier among the emulsions prepared in this work, so it appears that the thick and/or loosely-packed interface is disadvantageous for the stability of $\alpha$-tocopherol encapsulated in the emulsions. Because the P10S- and P20S-stabilized emulsions have droplet surfaces of similar density, it was expected that $\alpha$-tocopherol in the emulsion stabilized with P20S, which has a hydrophilic head size that is twice as large as that of
P10S, would be more stable than that in the P10S-stabilized emulsion; however, there was no significant difference in the initial decomposition rate of $\alpha$-tocopherol in these two emulsions $(p>0.05)$. P20S- and P23L-stabilized emulsions have interfacial membranes of similar thickness because the difference in the oxyethylene group number of the hydrophilic heads of P20S and P23L is only three, while the P20Sstabilized emulsion has a denser interfacial membrane than the P23L-stabilized emulsion, as described above. However, both of these emulsions showed very similar initial decomposition rates of $\alpha$-tocopherol, independent of the $\mathrm{pH}$ level. It was apparent that the thickness and/or density of the droplet surfaces did not affect the initial $\alpha$-tocopherol decomposition rate. In addition, considering the content (>90\%) of $\alpha$-tocopherol remaining after 21 days of storage, the variation in initial $\alpha$-tocopherol decomposition rates among the emulsions did not have much effect.

\subsection{Influence of Transition Metals on $\alpha$-Tocopherol} Degradation. The previous findings indicate that iron ions could be the direct or indirect reasons for the degradation of the several food components including lipids [23] and the precursors of vitamins such as carotenoids [24] because of their electron transfer reaction. The cation radicals could be formed by the interaction of iron ions with those food components. It means transition metals like iron could act as oxidizing agents. Therefore, in this study, iron ions were chosen as oxidants for studying the degradation of $\alpha$-tocopherol. The initial decomposition rates of $\alpha$-tocopherol in emulsions with iron were different from those of $\alpha$-tocopherol in iron-free emulsions. As shown in Table 1, the emulsions stabilized with different emulsifiers in the absence of iron had different initial $\alpha$-tocopherol decomposition rates, whereas little difference was observed in the initial $\alpha$-tocopherol decomposition rates for emulsions stabilized with different emulsifiers in the presence of iron. However, similar to the observation mentioned above, there was no significant difference in the $\alpha$-tocopherol content remaining in the emulsions after 21 days of storage $(p<0.05)$, which suggests that iron did not have an influence on the stability of $\alpha$-tocopherol in the emulsions (Figures 3 and 4).

Irrespective of the oxidative state of the iron, the initial decomposition rates of $\alpha$-tocopherol in emulsions in the presence of ferrous iron were not significantly different from those in emulsions stored with ferric iron $(p<0.05)$. In addition, when emulsions contained iron with the same oxidative state, they showed very similar initial decomposition rates of $\alpha$-tocopherol, regardless of the $\mathrm{pH}$ level. Although all of the emulsifiers used in this work were nonionic, the droplet surface charges of the emulsions were slightly negative and their values changed depending on the $\mathrm{pH}$ level $(-7.3,-6.8$, $-5.5,-9.4$, and $-1.7 \mathrm{mV}$ for P10L-, P10S-, P20S-, P23L-, and $\mathrm{P} 100 \mathrm{~S}$-stabilized emulsions at $\mathrm{pH} 7$, respectively, and around $-1.5 \mathrm{mV}$ at $\mathrm{pH} 3$ ). Because the droplet surfaces were more negatively charged at $\mathrm{pH} \mathrm{7,} \mathrm{except} \mathrm{those} \mathrm{in} \mathrm{the} \mathrm{P100S-}$ stabilized emulsion and could attract iron molecules to the surface of the emulsion droplets, it was expected that $\alpha$-tocopherol would be rapidly decomposed at $\mathrm{pH} 7$ because the 


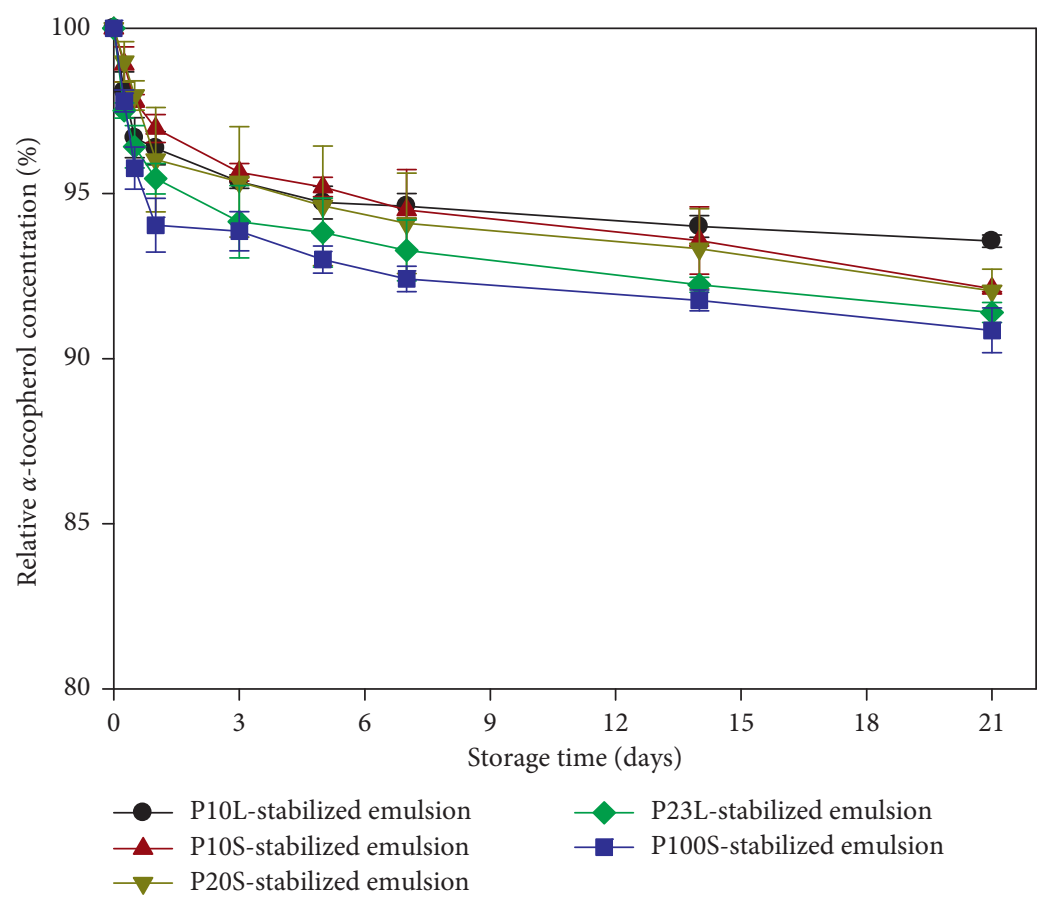

(a)

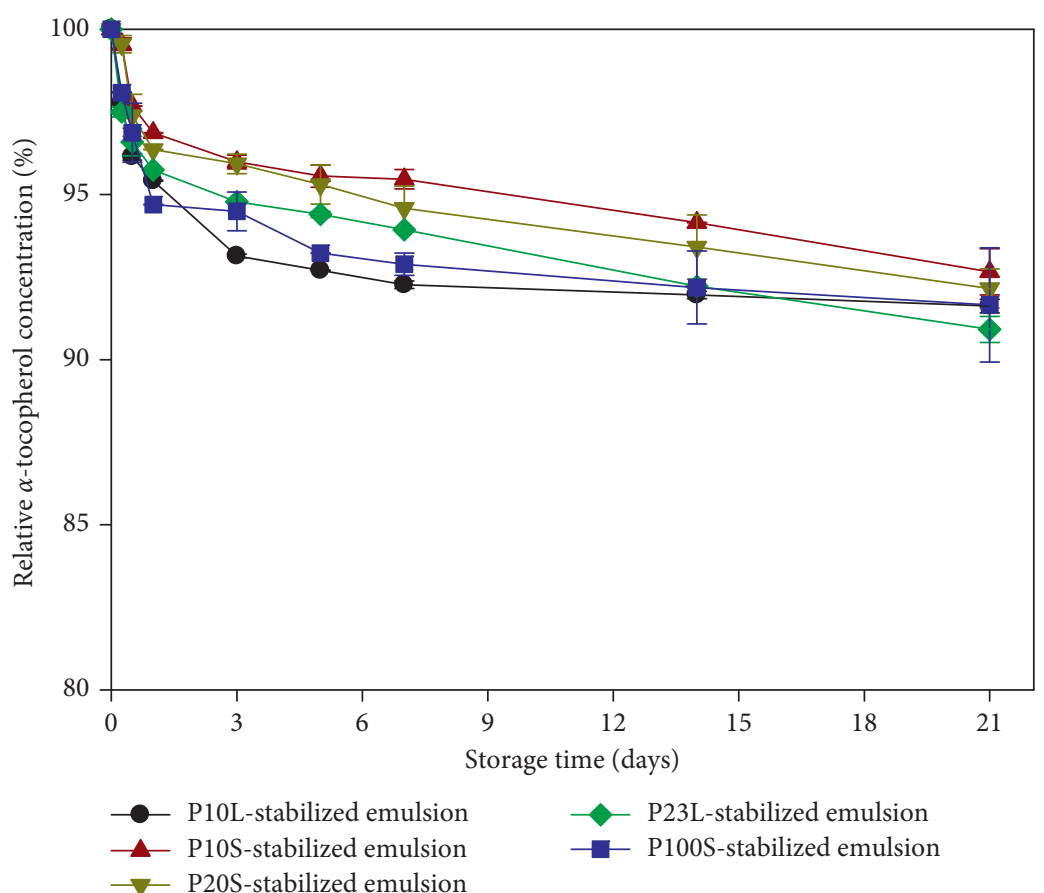

(b)

FIGURE 2: Change in concentration of $\alpha$-tocopherol in emulsions at $\mathrm{pH} 7$ (a) and 3 (b) stored at $25^{\circ} \mathrm{C}$. P10L, polyoxyethylene 10 lauryl ether; P10S, polyoxyethylene 10 stearyl ether; P20S, polyoxyethylene 20 stearyl ether; P23L, polyoxyethylene 23 lauryl ether; P100S, polyoxyethylene 100 stearyl ether.

iron molecules would accumulate around the more negatively charged droplet surfaces at this $\mathrm{pH}$ level. This suggests that the iron did not decompose $\alpha$-tocopherol by direct interaction at the interfacial membrane. Therefore, the stability of $\alpha$-tocopherol in emulsions was not influenced by the thickness and/or density of the droplet interfaces.
3.3. Influence of Radicals on $\alpha$-Tocopherol Degradation. AAPH may be a suitable material for studying the influence of radicals on the stability of $\alpha$-tocopherol encapsulated in emulsions because it is a self-generator of free radicals through spontaneous decomposition at room temperature. Quite different from the previous findings, in this case, the 
TABLE 1: The initial decomposition rate ( $\mathrm{mmol} / \mathrm{kg}$ emulsion/day) of $\alpha$-tocopherol in emulsions stabilized with emulsifiers having various sizes of hydrophilic and hydrophobic groups.

\begin{tabular}{|c|c|c|c|c|c|c|}
\hline & \multirow{2}{*}{ Environmental stress } & \multicolumn{5}{|c|}{ Emulsifier used for emulsion preparation } \\
\hline & & P10L & P10S & P20S & P23L & P100S \\
\hline \multirow{3}{*}{ pH 7} & No & ${ }^{\mathrm{AB}} 0.0185 \pm 0.0108^{\mathrm{ab}}$ & ${ }^{\mathrm{B}} 0.0034 \pm 0.0007^{\mathrm{b}}$ & ${ }^{\mathrm{B}} 0.0083 \pm 0.0094^{\mathrm{b}}$ & ${ }^{\mathrm{A}} 0.0098 \pm 0.0109^{\mathrm{ab}}$ & ${ }^{\mathrm{A}} 0.0266 \pm 0.0095^{\mathrm{a}}$ \\
\hline & errous iron & ${ }^{A B C} 0.0142 \pm 0.0003^{\mathrm{C}}$ & ${ }^{\mathrm{AB}} 0.0210 \pm 0.0000^{\mathrm{b}}$ & ${ }^{\mathrm{A}} 0.0250 \pm 0.0009^{\mathrm{a}}$ & ${ }^{\mathrm{A}} 0.0242 \pm 0.0028^{\mathrm{a}}$ & ${ }^{\mathrm{A}} 0.0211=$ \\
\hline & & ${ }^{\mathrm{A}} 0.0210 \pm 0.0093^{\mathrm{a}}$ & ${ }^{\mathrm{AB}} 0.0231 \pm 0.0028^{\mathrm{a}}$ & ${ }^{\mathrm{A}} 0.0215 \pm 0.0035^{\mathrm{a}}$ & ${ }^{\mathrm{A}} 0.0258 \pm 0.0323^{\mathrm{a}}$ & ${ }^{\mathrm{A}} 0.0230 \pm 0.0061^{\mathrm{a}}$ \\
\hline \multirow{3}{*}{$\mathrm{pH} 3$} & $\mathrm{~N}$ & $\mathrm{ABC}_{0.0173} \pm 0.0004^{\mathrm{b}}$ & ${ }^{\mathrm{B}} 0.0030 \pm 0.0000^{\mathrm{C}}$ & ${ }^{\mathrm{B}} 0.0034 \pm 0.0005^{\mathrm{c}}$ & ${ }^{\mathrm{A}} 0.0030 \pm 0.0005^{\mathrm{c}}$ & ${ }^{\mathrm{A}} 0.0188 \pm 0.0011^{\mathrm{a}}$ \\
\hline & Ferrou & ${ }^{\mathrm{A}} 0.0226 \pm 0.0017^{\mathrm{a}}$ & ${ }^{\mathrm{AB}} 0.0225 \pm 0.0021^{\mathrm{a}}$ & ${ }^{\mathrm{A}} 0.0250 \pm 0.0009^{\mathrm{a}}$ & ${ }^{\mathrm{A}} 0.0217 \pm 0.0042^{\mathrm{a}}$ & ${ }^{\mathrm{A}} 0.0107 \pm 0.0011^{\mathrm{b}}$ \\
\hline & Ferri & ${ }^{\mathrm{A}} 0.0231 \pm 0.0060^{\mathrm{a}}$ & ${ }^{\mathrm{A}} 0.0330 \pm 0.0407^{\mathrm{a}}$ & ${ }^{\mathrm{A}} 0.0264 \pm 0.0101^{\mathrm{a}}$ & ${ }^{\mathrm{A}} 0.0364 \pm 0.0467^{\mathrm{a}}$ & ${ }^{\mathrm{A}} 0.0198 \pm 0.0236^{\mathrm{a}}$ \\
\hline
\end{tabular}

P10L, polyoxyethylene 10 lauryl ether; P10S, polyoxyethylene 10 stearyl ether; P20S, polyoxyethylene 20 stearyl ether; P23L, polyoxyethylene 23 lauryl ether; P100S, polyoxyethylene 100 stearyl ether. The values with different small letter superscripts in the same row are significantly different $(p<0.05)$ by Duncan's multiple range test. The values with different capital letter superscripts in the same column are significantly different $(p<0.05)$ by Duncan's multiple range test.

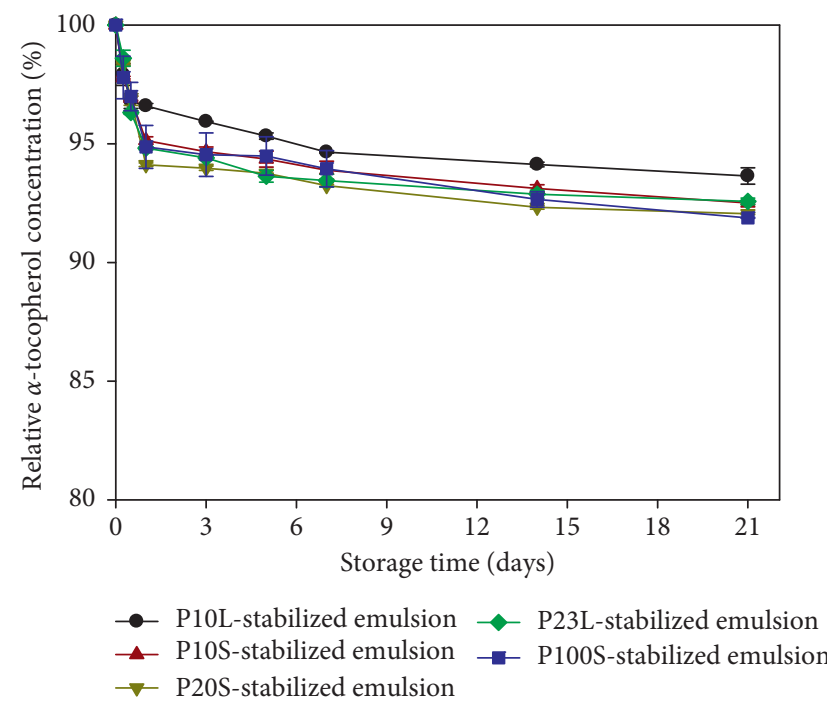

(a)

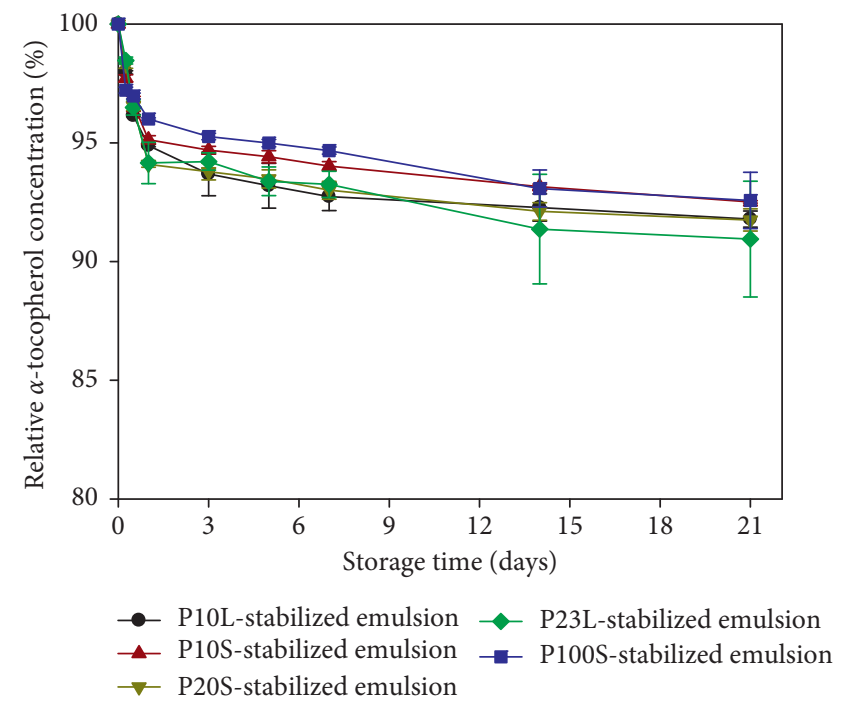

(b)

Figure 3: Change in concentration of $\alpha$-tocopherol in emulsions in the presence of ferrous iron at pH 7 (a) and 3 (b) stored at $25^{\circ} \mathrm{C}$. P10L, polyoxyethylene 10 lauryl ether; P10S, polyoxyethylene 10 stearyl ether; P20S, polyoxyethylene 20 stearyl ether; P23L, polyoxyethylene 23 lauryl ether; P100S, polyoxyethylene 100 stearyl ether.

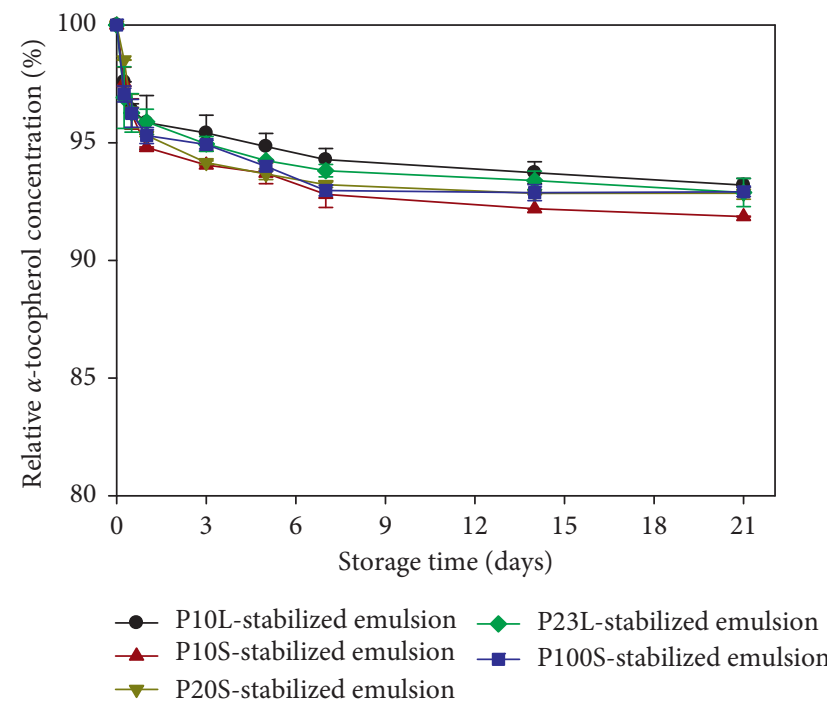

(a)

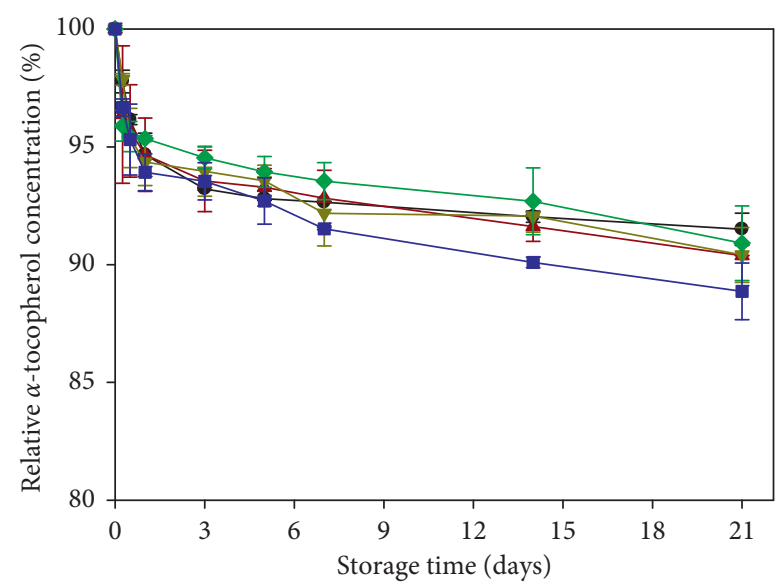

$\rightarrow$ P10L-stabilized emulsion $\multimap$ P23L-stabilized emulsion
$\neg$ P10S-stabilized emulsion $\rightarrow$ P100S-stabilized emulsion
$\rightarrow$ P20S-stabilized emulsion

(b)

FIGURE 4: Change in concentration of $\alpha$-tocopherol in emulsions in the presence of ferric iron at pH 7 (a) and 3 (b) stored at $25^{\circ} \mathrm{C}$. $\mathrm{P} 10 \mathrm{~L}$, polyoxyethylene 10 lauryl ether; P10S, polyoxyethylene 10 stearyl ether; P20S, polyoxyethylene 20 stearyl ether; P23L, polyoxyethylene 23 lauryl ether; P100S, polyoxyethylene 100 stearyl ether. 


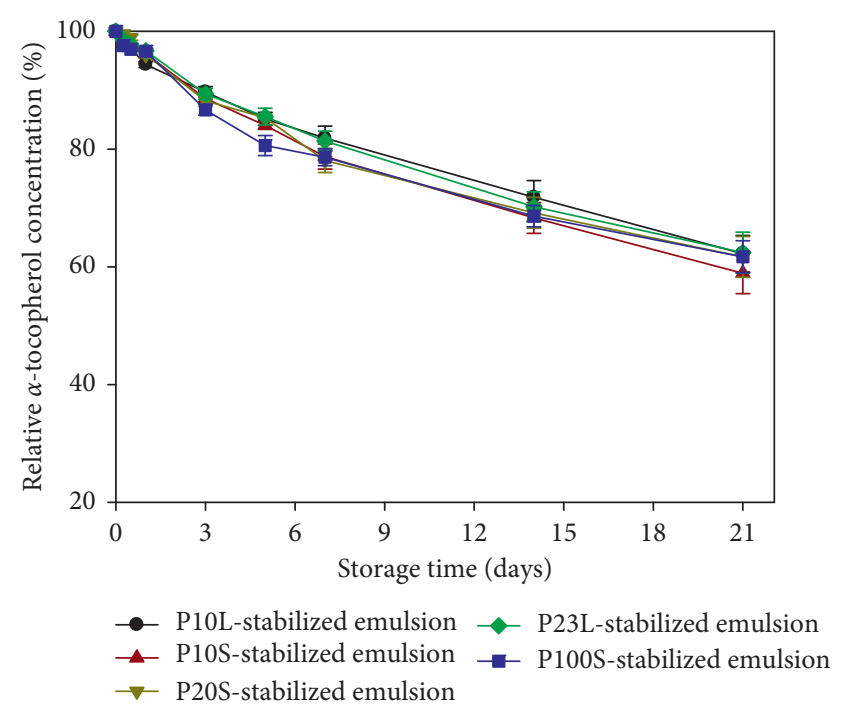

(a)

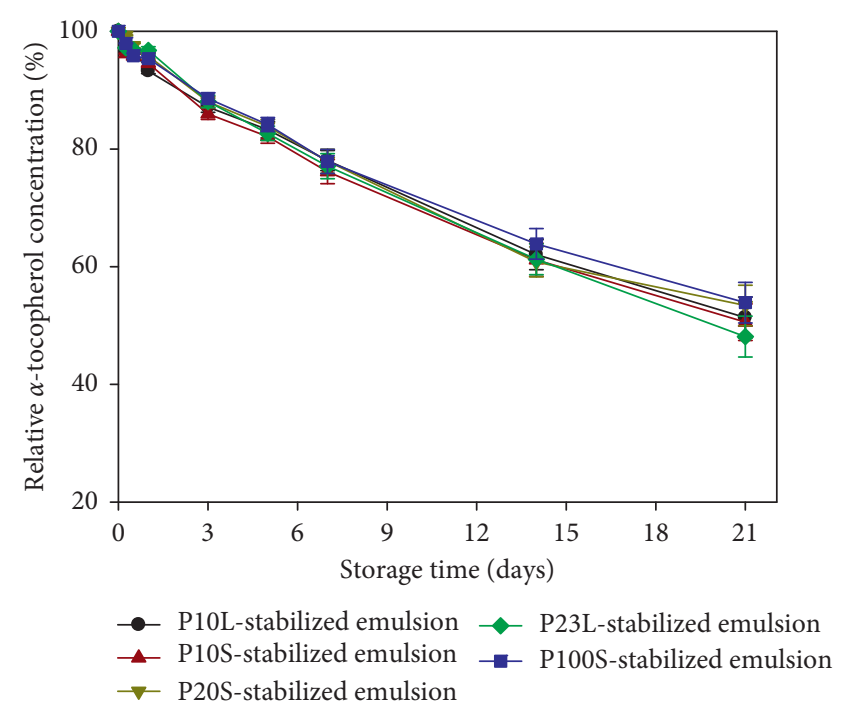

(b)

Figure 5: Change in concentration of $\alpha$-tocopherol in emulsions in the presence of radicals at $\mathrm{pH} 7$ (a) and 3 (b) stored at $25^{\circ} \mathrm{C}$. P10L, polyoxyethylene 10 lauryl ether; P10S, polyoxyethylene 10 stearyl ether; P20S, polyoxyethylene 20 stearyl ether; P23L, polyoxyethylene 23 lauryl ether; P100S, polyoxyethylene 100 stearyl ether.

TABLE 2: The decomposition rate $(k)$ of $\alpha$-tocopherol in emulsions stabilized with emulsifiers having various sizes of hydrophilic and hydrophobic groups in presence of radicals.

\begin{tabular}{|c|c|c|c|c|c|c|c|c|c|c|}
\hline & \multicolumn{10}{|c|}{ Emulsifier used for emulsion preparation } \\
\hline & \multicolumn{2}{|c|}{ P10L } & \multicolumn{2}{|c|}{ P10S } & \multicolumn{2}{|c|}{ P20S } & \multicolumn{2}{|c|}{$\mathrm{P} 23 \mathrm{~L}$} & \multicolumn{2}{|c|}{ P100S } \\
\hline & $k\left(\right.$ day $\left.^{-1}\right)$ & $r^{2}$ & $k\left(\right.$ day $\left.^{-1}\right)$ & $r^{2}$ & $k\left(\right.$ day $\left.^{-1}\right)$ & $r^{2}$ & $k\left(\right.$ day $\left.^{-1}\right)$ & $r^{2}$ & $k\left(\right.$ day $\left.^{-1}\right)$ & $r^{2}$ \\
\hline pH 7 & ${ }^{\mathrm{B}} 0.0218^{\mathrm{b}}$ & 0.989 & ${ }^{\mathrm{B}} 0.0252^{\mathrm{a}}$ & 0.984 & ${ }^{\mathrm{B}} 0.0235^{\mathrm{ab}}$ & 0.967 & ${ }^{\mathrm{B}} 0.0226^{\mathrm{ab}}$ & 0.985 & ${ }^{\mathrm{B}} 0.0230^{\mathrm{ab}}$ & 0.951 \\
\hline $\mathrm{pH} 3$ & ${ }^{\mathrm{A}} 0.0312^{\mathrm{bc}}$ & 0.996 & ${ }^{\mathrm{A}} 0.0319^{\mathrm{b}}$ & 0.994 & ${ }^{\mathrm{A}} 0.0310^{\mathrm{bc}}$ & 0.988 & ${ }^{\mathrm{A}} 0.0344^{\mathrm{a}}$ & 0.999 & ${ }^{\mathrm{A}} 0.0293^{\mathrm{c}}$ & 0.995 \\
\hline
\end{tabular}

P10L, polyoxyethylene 10 lauryl ether; P10S, polyoxyethylene 10 stearyl ether; P20S, polyoxyethylene 20 stearyl ether; P23L, polyoxyethylene 23 lauryl ether; P100S, polyoxyethylene 100 stearyl ether. The $\alpha$-tocopherol decomposition rate values with different small letter superscripts in the same row are significantly different $(p<0.05)$ by the Chow test. The $\alpha$-tocopherol decomposition rate values with different capital letter superscripts in the same column are significantly different $(p<0.05)$ by the Chow test.

$\alpha$-tocopherol concentration gradually decreased during the 21 -day storage period (Figure 5). The values of $k$ for $\alpha$-tocopherol in emulsions stored at $\mathrm{pH} 3$ ranged from 0.0293 to 0.0344 day $^{-1}$, and the values of $k$ for $\alpha$-tocopherol in emulsions stored at $\mathrm{pH} 7$ ranged from 0.0218 to $0.0252 \mathrm{day}^{-1}$ (Table 2). Although $\alpha$-tocopherol decomposed more quickly in acidic conditions than neutral conditions, the lack of correlation between the $k$ value and the properties (thickness and/or density) of the interfacial membranes suggests that the interfacial characteristics played little or no role in improving the stability of emulsified $\alpha$-tocopherol against radical-mediated oxidation.

During the design of this experiment, we expected that the properties of the interfacial membranes of oil droplets would affect the storage stability of $\alpha$-tocopherol incorporated in emulsions. Although there is a lack of information about the influence of the density of interfacial membranes on the oxidative stability of emulsified oils and the storage stability of encapsulated functional lipophilic compounds, according to previous studies, the emulsion interfacial thickness could be one of the important determinants of the oxidative stability of food emulsions [16]. Song et al. [11] reported that the storage stability of $\beta$-carotene in emulsions varied depending on the droplet interfacial thickness, and they also revealed that the droplet interfacial density may be a factor to consider for improving $\beta$-carotene stability. As described above, all of the emulsions analyzed in this study had different densities and thicknesses for their interfacial membranes. For example, the P100S-stabilized emulsion had the thickest interfacial membrane but its density was the lowest among the emulsions, whereas the P10L- and P10S-stabilized emulsions had the opposite properties. The stability of $\alpha$-tocopherol in the emulsions greatly depended on the environmental conditions surrounding the emulsion droplets, and the denseness and/or thickness of the interfacial membrane of the oil droplets did not play a crucial role in improving the stability of the encapsulated $\alpha$-tocopherol. In conclusion, our findings, together with those of previous studies, suggested that the data are still insufficient to generalize the influence of droplet interface characteristics on the oxidative stability of emulsified oils 
and the chemical stability of encapsulated oil-soluble components.

\section{Data Availability}

The data used to support the findings of this study are available from the corresponding author upon request.

\section{Conflicts of Interest}

The authors declare that they have no conflicts of interest.

\section{Acknowledgments}

This study was supported by the Research Program funded by Seoul National University of Science and Technology.

\section{References}

[1] E. N. Frankel, "Lipid oxidation: mechanisms, products and biological significance," Journal of the American Oil Chemists' Society, vol. 61, no. 12, pp. 1908-1917, 1984.

[2] D. J. McClements, E. A. Decker, and J. Weiss, "Emulsionbased delivery systems for lipophilic bioactive components," Journal of Food Science, vol. 72, no. 8, pp. R109-R124, 2007.

[3] D. J. McClements, "Emulsion design to improve the delivery of functional lipophilic components," Annual Review of Food Science and Technology, vol. 1, no. 1, pp. 241-269, 2010.

[4] E. N. Frankel, "Antioxidants in lipid foods and their impact on food quality," Food Chemistry, vol. 57, no. 1, pp. 51-55, 1996.

[5] D. J. McClements, Food Emulsions: Principles, Practices, and Techniques, CRC Press, Boca Raton, FL, USA, 2nd edition, 2005.

[6] H. D. Silva, M. A. Cerqueira, B. W. S. Souza et al., "Nanoemulsions of $\beta$-carotene using a high-energy emulsificationevaporation technique," Journal of Food Engineering, vol. 102, no. 2, pp. 130-135, 2011.

[7] S. W. Han, H. Y. Song, T. W. Moon, and S. J. Choi, "Influence of emulsion interfacial membrane characteristics on Ostwald ripening in a model emulsion," Food Chemistry, vol. 242, pp. 91-97, 2018.

[8] B. Chen, D. J. McClements, and E. A. Decker, "Role of continuous phase anionic polysaccharides on the oxidative stability of Menhaden oil-in-water emulsions," Journal of Agricultural and Food Chemistry, vol. 58, no. 6, pp. 37793784, 2010.

[9] H. Faraji, D. J. McClements, and E. A. Decker, "Role of continuous phase protein on the oxidative stability of fish oilin-water emulsions," Journal of Agricultural and Food Chemistry, vol. 52, no. 14, pp. 4558-4564, 2004.

[10] C. Qian, E. A. Decker, H. Xiao, and D. J. McClements, "Physical and chemical stability of $\beta$-carotene-enriched nanoemulsions: influence of $\mathrm{pH}$, ionic strength, temperature, and emulsifier type," Food Chemistry, vol. 132, no. 3, pp. 1221-1229, 2012.

[11] H. Y. Song, T. W. Moon, and S. J. Choi, "Storage stability of $\beta$-carotene in model beverage emulsions: implication of interfacial thickness," European Journal of Lipid Science and Technology, vol. 120, no. 9, article 1800127, 2018.

[12] D. J. McClements, "Nanoemulsion-based oral delivery systems for lipophilic bioactive components: nutraceuticals and pharmaceuticals," Therapeutic Delivery, vol. 4, no. 7, pp. 841-857, 2013.
[13] T. G. Mason, J. N. Wilking, K. Meleson, C. B. Chang, and S. M. Graves, "Nanoemulsions: formation, structure, and physical properties," Journal of Physics: Condensed Matter, vol. 18, no. 41, pp. R635-R666, 2006.

[14] D. J. McClements and J. Rao, "Food-grade nanoemulsions: formulation, fabrication, properties, performance, biological fate, and potential toxicity," Critical Reviews in Food Science and Nutrition, vol. 51, no. 4, pp. 285-330, 2011.

[15] J. R. Mancuso, D. J. McClements, and E. A. Decker, "Ability of iron to promote surfactant peroxide decomposition and oxidize $\alpha$-tocopherol," Journal of Agricultural and Food Chemistry, vol. 47, no. 10, pp. 4146-4149, 1999.

[16] M. P. C. Silvestre, W. Chaiyasit, R. G. Brannan, D. J. McClements, and E. A. Decker, "Ability of surfactant headgroup size to alter lipid and antioxidant oxidation in oilin-water emulsions," Journal of Agricultural and Food Chemistry, vol. 48, no. 6, pp. 2057-2061, 2000.

[17] Y. Yang, E. A. Decker, H. Xiao, and D. J. McClements, "Enhancing vitamin E bioaccessibility: factors impacting solubilization and hydrolysis of $\alpha$-tocopherol acetate encapsulated in emulsion-based delivery systems," Food and Function, vol. 6, no. 1, pp. 84-97, 2015.

[18] G. C. Chow, "Tests of equality between sets of coefficients in two linear regressions," Econometrica, vol. 28, no. 3, pp. 591-605, 1960.

[19] D. J. McClements, "Ultrasonic determination of depletion flocculation in oil-in-water emulsions containing a non-ionic surfactant," Colloids and Surfaces A-Physicochemical and Engineering Aspects, vol. 90, no. 1, pp. 24-35, 1994.

[20] USEPA, Estimation Program Interface Suite (TM), Ver. 4.11, United States Environmental Protection Agency, Washington, DC, USA, 2012.

[21] J. F. I. Gregory, "Vitamins," in Fennema's Food Chemistry, S. Damodaran, K. L. Parkin, and O. R. Fennema, Eds., CRC Press, Boca Raton, FL USA, 4 edition, 2008.

[22] J. A. Heydinger and D. K. Nakhasi, "Medium chain triacylglycerols," Journal of Food Lipids, vol. 3, no. 4, pp. 251257, 1996.

[23] E. Choe and D. B. Min, "Mechanisms and factors for edible oil oxidation," Comprehensive Reviews in Food Science and Food Safety, vol. 5, no. 4, pp. 169-186, 2006.

[24] C. S. Boon, D. J. McClements, J. Weiss, and E. A. Decker, "Factors influencing the chemical stability of carotenoids in foods," Critical Reviews in Food Science and Nutrition, vol. 50, no. 6, pp. 515-532, 2010. 

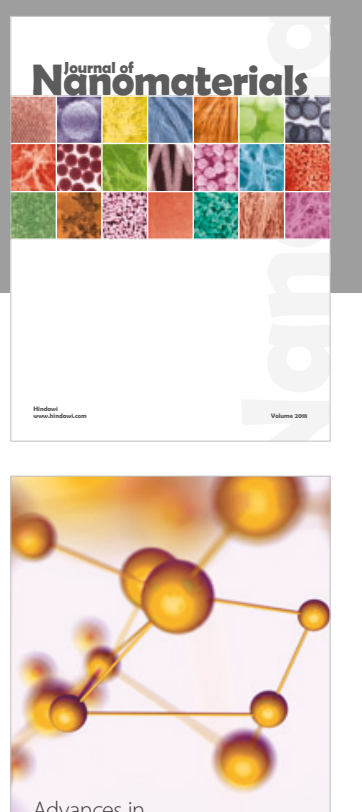

Physical Chemistry
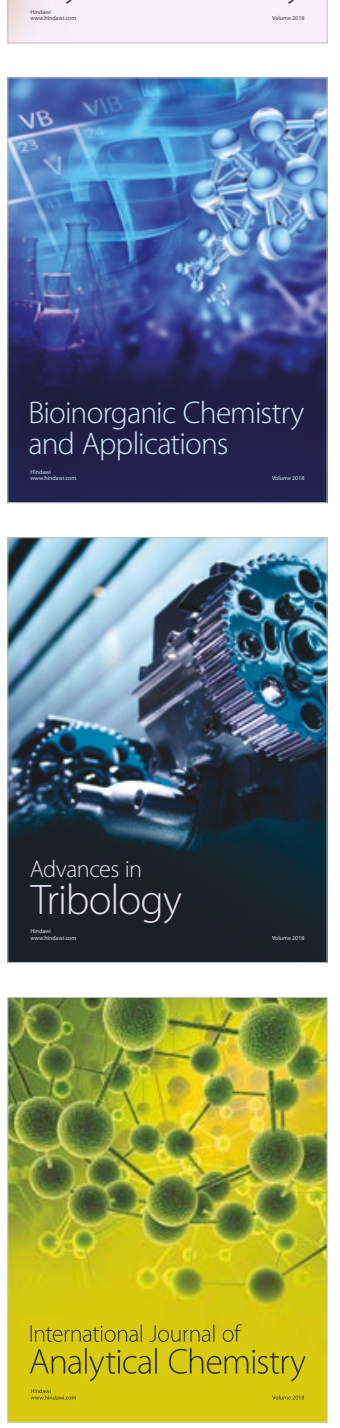

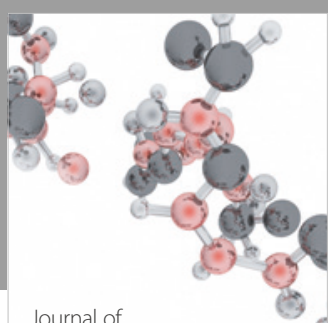

Analytical Methods

in Chemistry

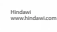

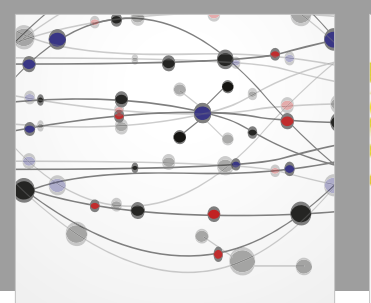

The Scientific World Journal

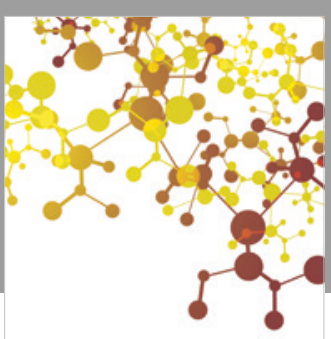

Journal of

Applied Chemistry
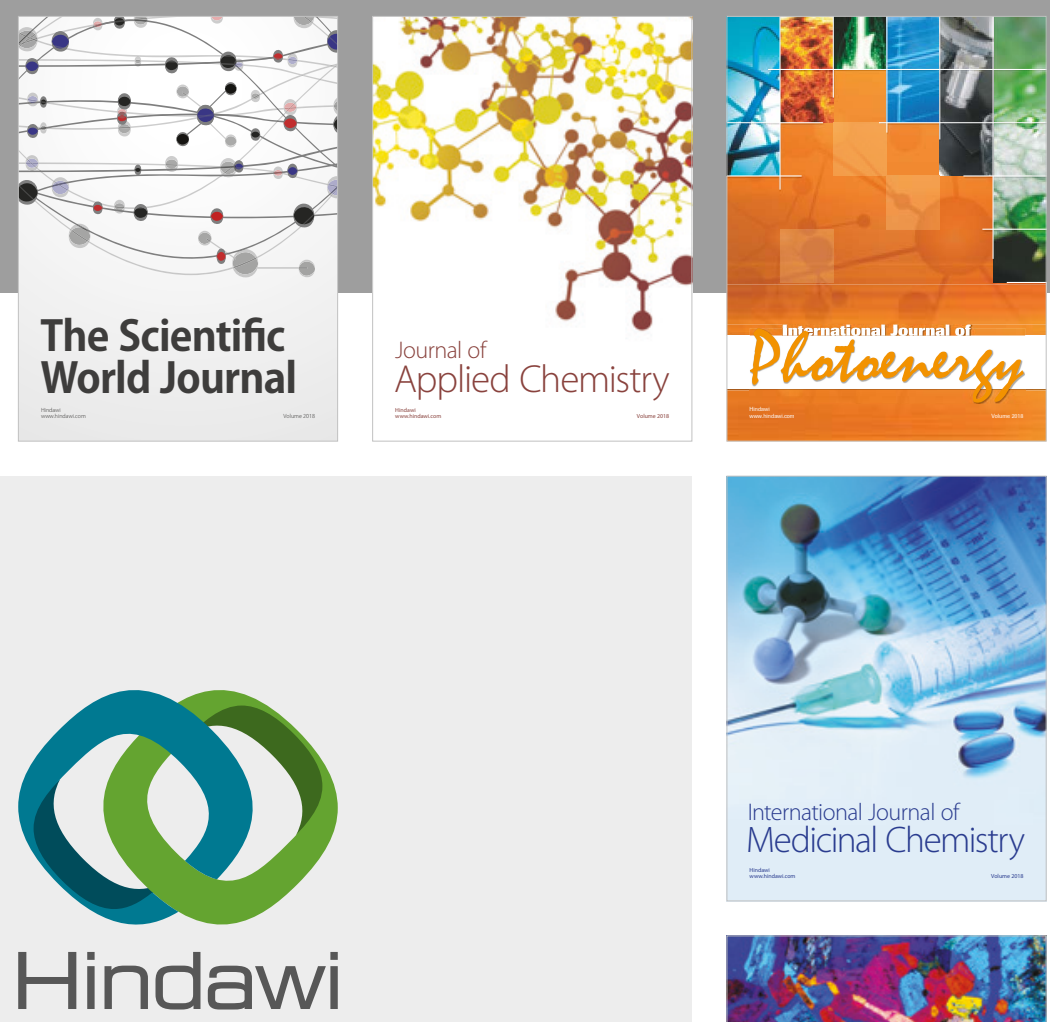

Submit your manuscripts at

www.hindawi.com
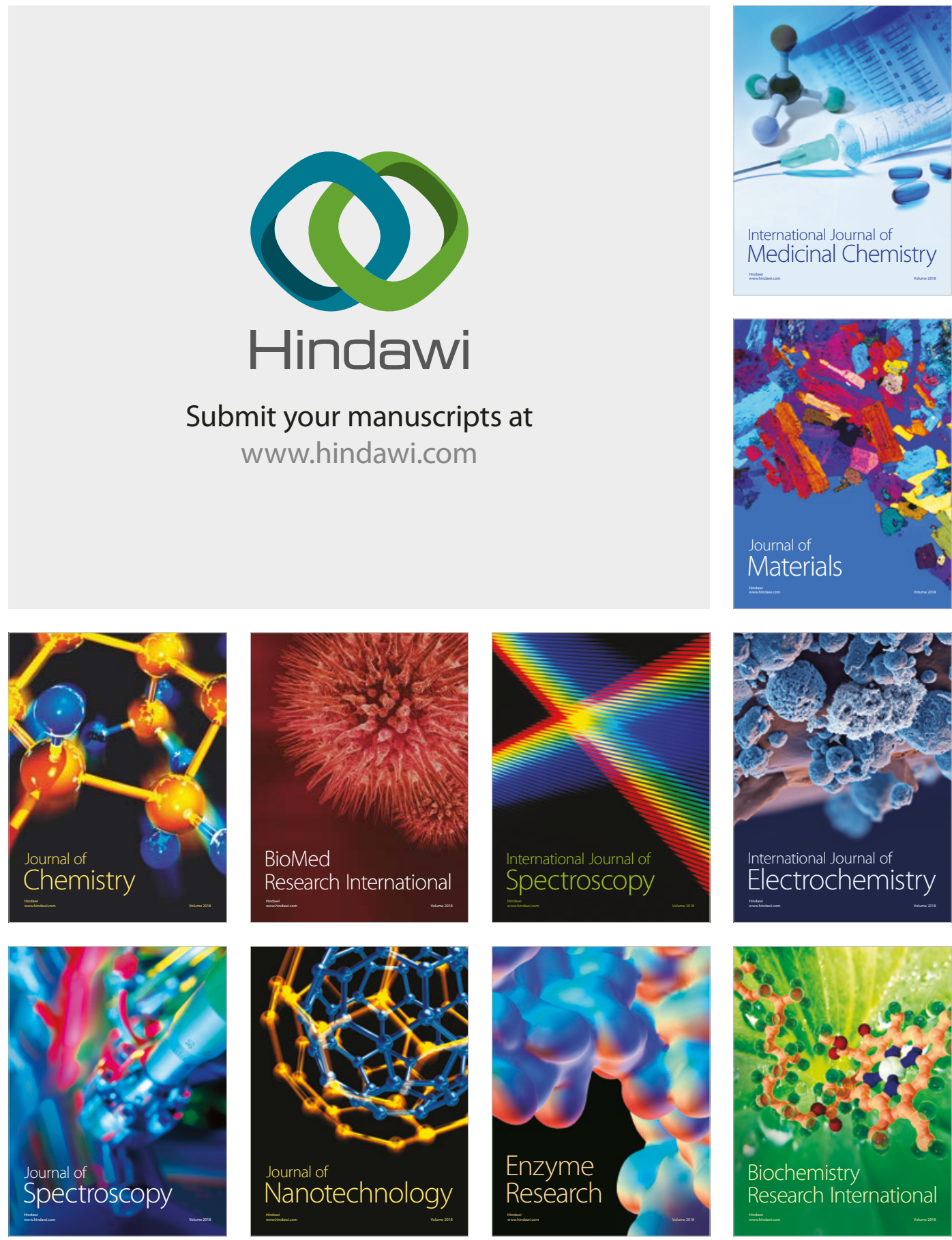
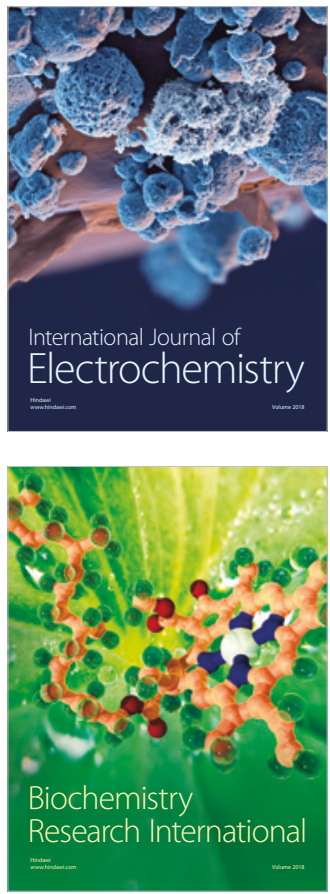